\title{
The influence of preoperative biopsy on the surgical method in breast cancer patients: a single-center experience of 3,966 cases in China
}

\author{
Rongyue Teng^, Qun Wei, Jichun Zhou, Mingjun Dong, Lidan Jin, Wenxian Hu, Jida Chen, \\ Linbo Wang, Wenhe Zhao
}

Department of Surgical Oncology, Sir Run Run Shaw Hospital, College of Medicine, Zhejiang University, Hangzhou, China

Contributions: (I) Conception and design: W Zhao; (II) Administrative support: W Zhao; (III) Provision of study materials or patients: All authors; (IV) Collection and assembly of data: All authors; (V) Data analysis and interpretation: All authors; (VI) Manuscript writing: All authors; (VII) Final approval of manuscript: All authors.

Correspondence to: Wenhe Zhao. Department of Surgical Oncology, Sir Run Run Shaw Hospital, College of Medicine, Zhejiang University, Hangzhou 310016, China. Email: whzhao@zju.edu.cn.

\begin{abstract}
Background: The National Surgical Adjuvant Breast and Bowel Project (NSABP) B32 trial reported that the detection rate of sentinel lymph nodes by core needle biopsy (CNB) is higher than that by segmental resection. However, there are few reports regarding the detection rate of sentinel lymph nodes by vacuumassisted breast biopsy (VABB). Therefore, we analyzed the impact of preoperative biopsy methods on the surgical modes of 3,966 patients with breast cancer in our center.

Methods: In total, 3,966 female breast cancer patients [clinical tumor node metastasis (TNM) stage I-III] were enrolled in this study. Preoperative pathological diagnosis methods included fine needle aspiration (FNA) biopsy, CNB, excision biopsy, and VABB. According to the time of diagnosis. The data were analysis by chi square test, variance analysis and the Kaplan-Meier time series in SPSS 22.0.
\end{abstract}

Results: There was a decrease in the number of patients that underwent excision biopsy (7.3\% to $2.7 \%$ ) and intraoperative freezing $(89.4 \%$ to $28.9 \%)$ over time, while CNB exhibited an increasing trend $(1.6 \%$ to $55.3 \%$ ). The positive rates of VABB, CNB, excision biopsy, and FNA were $99.5 \%, 97.1 \%, 97.9 \%$, and $82.2 \%$, respectively, and the false negative rates were $0 \%, 1.8 \%, 0.34 \%$, and $8.9 \%$, respectively. The overall breast-conserving rate was $36.7 \%$, while the breast-conserving rate for VABB was $57.1 \%$. The axillary sentinel lymph node biopsy rate of cN0 patients was $48.3 \%$, and the intraoperative frozen group (36.7\%) and excision biopsy group (39.5\%) were lower than the CNB (57.1\%) and VABB (77.9\%) groups. Until December 2019, there were 350 cases with tumor recurrence or metastasis. The methods of biopsy were not correlated to the cumulative survival time.

Conclusions: Changes to the diagnosis and treatment of breast cancer has a profound impact on the method of tumor biopsy. VABB biopsy offers advantages such as accurate diagnosis, a greater volume of tissue taken at one time, minimally invasive and repeatable, and does not affect the surgical approach and prognosis of patients. It will gradually become the primary method of preoperative pathological evaluation of breast cancer.

Keywords: Breast cancer; core needle biopsy (CNB); excision; vacuum-assisted breast biopsy (VABB); overall survival

Submitted Dec 01, 2020. Accepted for publication Feb 26, 2021.

doi: $10.21037 / \mathrm{gs}-21-7$

View this article at: http://dx.doi.org/10.21037/gs-21-7

\footnotetext{
$\wedge$ ORCID: 0000-0003-2621-7577.
} 


\section{Introduction}

Breast cancer is the most common malignancy in women. With the progress of research, the methods of tumor treatment have become increasingly standardized. Individualized treatment, such as breast-conserving surgery, axillary sentinel lymph node biopsy, and new adjuvant therapy, as well as patient participation in the treatment decision, requires more precise preoperative tumor biopsy. The traditional methods of preoperative tumor biopsy, including resection biopsy, core needle biopsy (CNB), or fine needle aspiration (FNA) biopsy, have been shown to be safe and have no impact on the prognosis of patients (1-4). With the development of biopsy diagnosis technology and the increased popularity of the newly developed vacuumassisted breast biopsy (VABB) such as Mammotome biopsy (Devicor ${ }^{\circledR}$ Medical Products, USA) and Cassi biopsy (Scion Medical Technologies, USA), these methods will play an increasingly important role in the diagnosis of breast cancer. However, there is no long-term research reporting on whether this novel biopsy mode is consistent with the traditional biopsy methods in terms of its surgical procedure and impact on the prognosis of breast cancer patients. This study reviewed the breast cancer biopsy data in our center for 20 years, summarized the differences between the novel preoperative biopsy $\mathrm{VABB}$ approach and the traditional biopsy techniques, and discussed its influence on treatment decisions and prognosis.

We present the following article in accordance with the STROBE reporting checklist (available at http://dx.doi. org/10.21037/gs-21-7).

\section{Methods}

From January 1998 to May 2019, a total of 5,109 newly treated breast cancer patients were admitted to our center. The exclusion criteria flow chart is shown in Figure 1. There were 3,966 cases with tumor node metastasis (TNM) stage I-III female breast cancer patients undergoing radical surgery for the first time. Preoperative pathological diagnosis methods included FNA, CNB, excision biopsy, VABB, and Cassi biopsy. If the pathological diagnosis of the biopsy was clinically suspicious, the tumor could not be confirmed by puncture, and the patient refuses biopsy, further resection biopsy and intraoperative frozen section was performed. According to the biopsy results, breast cancer can be divided into the following: confirmed breast cancer, undetermined diagnosis (including suspicious tumors, atypical hyperplasia, undetermined diagnosis such as undetermined papillary tumors or only a small amount of dysplasia), false negative puncture sample, or frozen section without tumor tissue. The diagnosis was reconfirmed by freezing. Resected specimens from other hospitals were reconfirmed by the pathology department in our hospital.

\section{Statistical analysis}

SPSS 22.0 software (IBM, USA) was used for statistical analysis. The chi square test and variance analysis were used to compare the different characteristics between all the groups. The Kaplan-Meier time series test was used to analyze survival time of different biopsy groups. Log rank was used to compare the survival time, and $\mathrm{P}<0.05$ was considered as the statistically significant standard.

This study was approved by Medical Ethics Committee of Sir Run Run Shaw Hospital (Approval NO: 2020-61801). All procedures performed in this study involving human participants were in accordance with the Declaration of Helsinki (as revised in 2013). Individual consent for this retrospective analysis was waived.

\section{Results}

There were 3,966 cases analyzed in this study, including eight cases of simultaneous bilateral cancer and nine cases of isochronous bilateral cancer. Ninety-four cases were associated with other malignant tumors, including 44 cases of thyroid cancer, 27 cases of lung cancer, nine cases of colorectal cancer, three cases of ovarian cancer, three cases of more than three primary cancers, and eight cases of other malignant tumors. The median age of included patients was 51 years (range, 21-89 years). Notably, there were 2,071 cases with breast tumors on the left side and 1,895 cases with breast tumors on the right side. Furthermore, there were 2,073 premenopausal cases, 1,811 menopausal cases, and 82 cases of unknown menstrual state.

According to the time of diagnosis, the results can be divided into five periods: 1998 to 2002, 2003 to 2007, 2008 to 2012, 2013 to 2017, and 2018 to May 2019. Table 1 shows the development of tumor biopsy methods among these five time periods. Over time, there was a decrease in local resection $(7.3 \%$ to $2.7 \%)$ and intraoperative frozen section (89.4\% to $28.9 \%$ ), while CNB diagnosis increased every year $(1.6 \%$ to $55.3 \%)$. In 2009 , the proportion of breast cancer diagnosed by VABB increased from $0.3 \%$ to $13.0 \%$, 


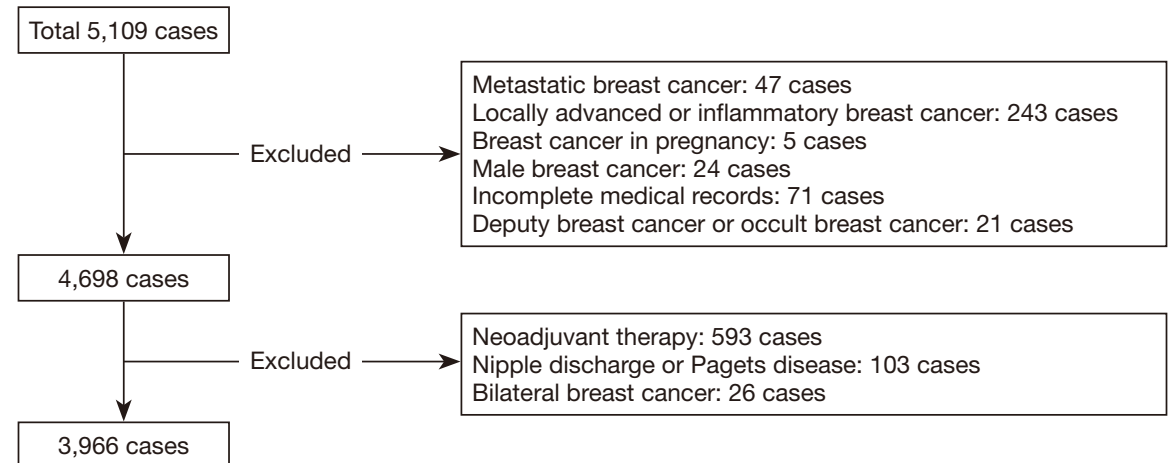

Figure 1 The flow chart for exclusion criteria of 5,109 newly treated breast cancer patients. There were 3,966 cases with TNM stage I-III female breast cancer patients were included in this study.

Table 1 Development of preoperative tumor biopsy techniques in five time periods

\begin{tabular}{|c|c|c|c|c|c|c|c|}
\hline $\begin{array}{l}\text { Characteristics and } \\
\text { biopsy techniques }\end{array}$ & CNB & $\begin{array}{l}\text { Intraoperative } \\
\text { frozen resection }\end{array}$ & Local resection & VABB & FNA & Total & $P$ value \\
\hline Median age & 52 & 50 & 45.5 & 48 & 51 & & \\
\hline \multicolumn{7}{|c|}{ Development of preoperative biopsy techniques in five time periods } & $<0.001$ \\
\hline 1998-2002 & $2(1.6)$ & $110(89.4)$ & $9(7.3)$ & 0 & $2(1.6)$ & 123 & \\
\hline 2008-2012 & 228 (31.9) & 419 (58.6) & $51(7.1)$ & $6(0.8)$ & $11(1.5)$ & 715 & \\
\hline 2013-2017 & 1,043 (53.9) & $742(38.4)$ & $66(3.4)$ & $70(3.6)$ & $13(0.7)$ & 1,934 & \\
\hline 2018-2019 & $478(55.3)$ & 250 (28.9) & $23(2.7)$ & $112(13.0)$ & $1(0.1)$ & 864 & \\
\hline $\begin{array}{l}\text { Unconfirmed } \\
\text { diagnosis }\end{array}$ & $20(1.1)$ & $30(1.7)$ & 0 & $1(0.5)$ & $4(8.9)$ & 55 & \\
\hline False negative & $33(1.8)$ & $6(0.3)$ & 0 & 0 & $4(8.9)$ & 43 & \\
\hline \multicolumn{7}{|c|}{ Tumor biopsy influence on surgical approach } & $<0.001$ \\
\hline \multicolumn{8}{|l|}{ Mastectomy } \\
\hline Yes & 1,115 & 1,177 & 99 & 81 & 36 & 2,508 & \\
\hline No & 700 & 570 & 71 & 108 & 9 & 1,458 & \\
\hline \multicolumn{8}{|c|}{ Sentinel lymph node biopsy ${ }^{*} n=3,598$} \\
\hline
\end{tabular}

Data are $\mathrm{n}$ or $\mathrm{n}(\%) .{ }^{*}$, cN0 or cN1 but FNA negative. VABB, vacuum-assisted breast biopsy; FNA, fine needle aspiration; CNB, core needle biopsy; SLNB, sentinel lymph node biopsy. 


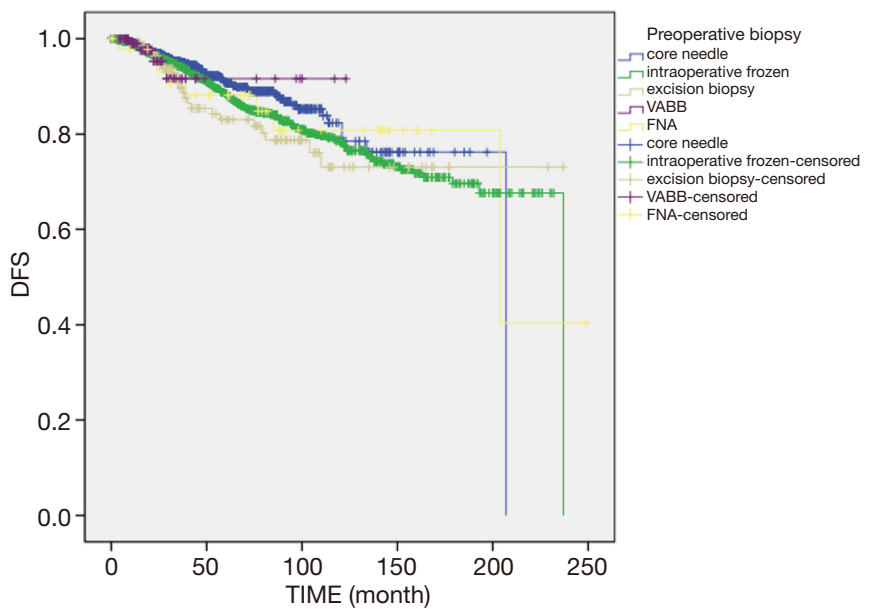

Figure 2 The biopsy method and the disease-free survival time. The 1-, 3-, 5-, 7-, 10-, 15-year tumor free survival was $98.8 \%$, $94.3 \%, 89.2 \%, 85.6 \%, 79.9 \%$ and $71.8 \%$ respectively. Log rank survival analysis showed that tumor biopsy is not correlated with tumor free survival $\left(\chi^{2}=2.668, \mathrm{P}=0.102\right)$. $\mathrm{CNB}$, core needle biopsy; $\mathrm{VABB}$, vacuum-assisted breast biopsy; FNA, fine needle aspiration.

and the difference was statistically significant $\left(\chi^{2}=606.479\right.$, $\mathrm{P}=0.000$, Table 1).

The final pathological diagnosis rate of five preoperative biopsy methods is shown in Table 1. The diagnosis rate of VABB was $99.5 \%$ (188/189), CNB was $97.1 \%(1,762 / 1,815)$, local resection was $97.9 \%(1,711 / 1,747)$, and FNA was $82.2 \%$ (37/45), while the false negative of these methods was as follows: $1.8 \%(33 / 1,815), 0.34 \%(6 / 1,747), 0 \%$ $(0 / 170)$ and $8.9 \%(4 / 45)$, respectively, and the difference was statistically significant $\left(\chi^{2}=72.423, \mathrm{P}=0.000\right)$. It is suggested that VABB is the most effective method for the preoperative diagnosis of breast cancer, and CNB can replace local resection biopsy. In the ductal carcinoma in situ (DCIS) group, the false negative rates of CNB and local resection biopsy were 5.1\% (5/99) and 2.3\% (5/219), respectively, while the ratio of tumors that could not be confirmed in the CNB group was 8.1\% (8/99), and was $9.1 \%$ $(20 / 219)$ in the local resection biopsy group $\left(\chi^{2}=24.792\right.$, $\mathrm{P}=0.002)$. For invasive cancers, the false negative rate of the CNB and local resection biopsy groups was $1.3 \%$ $(21 / 1,593)$ and $0 \%(0 / 1,351)$, respectively, and the ratio of tumors that could not be confirmed in these groups was $0.3 \%(5 / 1,593)$ and $0.4 \%(6 / 1,351)$, respectively $\left(\chi^{2}=66.853\right.$, $\mathrm{P}=0.000$ ). Moreover, in the $\mathrm{T} 1$ group (tumor $\leq 2 \mathrm{~cm}$ ), the false negative rates of $\mathrm{CNB}$, local resection biopsy, and FNA were $2.3 \%(21 / 924), 0.3 \%(3 / 1,057)$, and $9.1 \%(2 / 22)$, respectively, and the undetermined rates of tumor pathology were $1.3 \%(12 / 924), 1.8 \%(19 / 1,057)$, and $13.6 \%(3 / 22)$, respectively $\left(\chi^{2}=56.936, \mathrm{P}=0.000\right)$

In this study, the overall breast-conserving rate was $36.7 \%$. The correlation between tumor biopsy and the breast-conserving rate is shown in Table 1. The breastconserving rate of breast cancer confirmed by VABB was $57.1 \%$ (108/189), including 134 cases of incidental breast cancer [54.5\% (73/134) of breast conserving rate] and 55 cases of breast cancer confirmed by VABB biopsy (63.6\% of breast conserving rate). The breast-conserving rate in the local resection group was $41.7 \%$, which was higher than the overall breast-conserving rate. It has been suggested that VABB and preoperative local tumor resection do not affect breast-conserving surgery. The immediate breast reconstruction rate in 69 patients who underwent mastectomy was $2.8 \%$. The reconstruction rate in the VABB group $11.1 \%(9 / 81)$ was the highest, the FNA group was $0 \%$, and the $\mathrm{CNB}$ group was $3.5 \%$ (Table 1).

The results showed that 3,600 cases of axillary cN0 or cN1 were preoperatively evaluated, however no lymph node metastasis was confirmed by FNA biopsy. 1,739 cases $(48.3 \%, 1,739 / 3,600)$ of axillary sentinel lymph node biopsy were performed. The rate of sentinel lymph node biopsy in the frozen (36.7\%) and the local resection (39.5\%) groups was lower than that in the CNB (57.1\%) and VABB (77.9\%) groups. The successful rate of sentinel lymph node biopsy was $75.2 \%(1,307 / 1,739)$, which was not affected by the biopsy method (Table 1). The sentinel lymph node metastasis rate was $21.2 \%(368 / 1,739)$ in patients with cN0 or $\mathrm{cN} 1$ (negative axillary puncture).

Until December 2019, there were 350 cases of tumor recurrence or metastasis, 149 cases of death, and 47 cases that were loss to follow-up. The end points of the study were tumor recurrence, death due to other diseases, or recurrent breast cancer of the contralateral breast. The 1-, 3-, 5-, 7-, 10-, and 15-year tumor-free survival was 98.8\%, $94.3 \%, 89.2 \%, 85.6 \%, 79.9 \%$, and $71.8 \%$, respectively. Log rank survival analysis showed that tumor biopsy was not correlated with tumor-free survival $\left(\chi^{2}=2.668, \mathrm{P}=0.102\right)$ (Figure 2). The cumulative survival time was calculated based on the patient's death. The total 1-, 3-, 5-, 7-, 10-, and 15 -year cumulative survival times were $99.9 \%, 98.2 \%$, $96.0 \%, 93.4 \%, 90.2 \%$, and $83.6 \%$, respectively. There was no correlation between the biopsy method and the cumulative survival time $\left(\chi^{2}=0.364, \mathrm{P}=0.546\right)$ (Figure 3). 


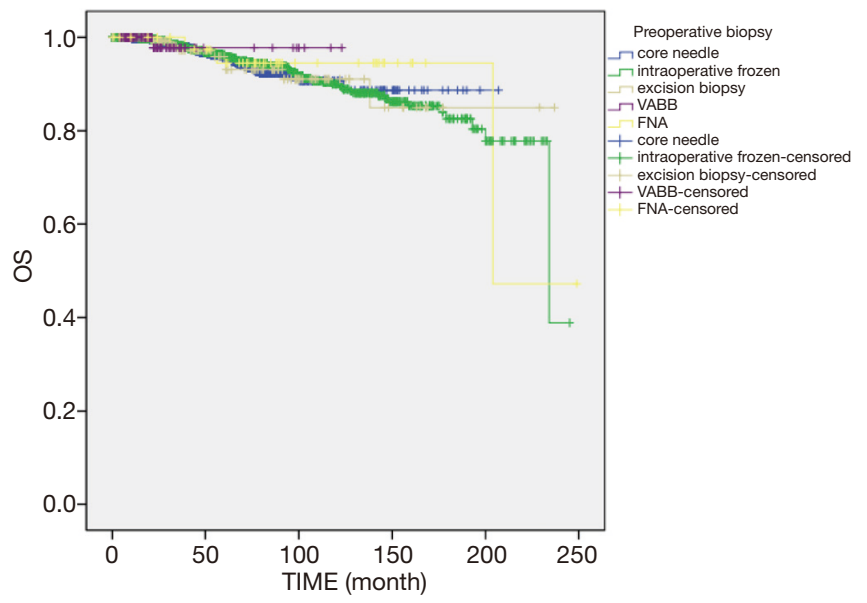

Figure 3 The biopsy method and the Overall survival time. The total 1-, 3-, 5-, 7-, 10-, 15-year cumulative survival time was $99.9 \%, 98.2 \%, 96.0 \%, 93.4 \%, 90.2 \%$ and $83.6 \%$ respectively. There was no correlation between the biopsy method and the cumulative survival time $\left(\chi^{2}=0.364, \mathrm{P}=0.546\right)$. $\mathrm{CNB}$, core needle biopsy; VABB, vacuum-assisted breast biopsy; FNA, fine needle aspiration.

\section{Discussion}

The purpose of this study was to review and analyze the preoperative biopsy data of breast cancer patients in our center from the past 20 years, and to observe the changes in preoperative pathological diagnosis modes for these patients. We also sought to examine the influence of VABB on treatment and prognosis. Previously, tumor resection biopsy (including intraoperative frozen section) was the main preoperative pathological diagnosis method for breast cancer. With the development of imaging and pathology, especially the understanding of molecular diagnosis of breast cancer, breast-conserving surgery, sentinel lymph node biopsy, axillary-conserving surgery, and new adjuvant treatment were carried out individually.

The proportion of tumor resection as a breast cancer diagnostic method has exhibited a decreasing trend over time, and is only used as a secondary option when $\mathrm{CNB}$ results are uncertain or inconsistent with imaging results (5). Technically, tumor resection biopsy is not a biopsy, but a partial mastectomy. After local tumor resection or intraoperative frozen section, the abnormal drainage of breast lymph nodes, together with the concerns of patients and doctors, led to a decrease in the rate of axillary sentinel lymph node biopsy. According to the data from our center, tumor resection biopsy (including intraoperative frozen section) has decreased from 96.7\% (119/123) before 2002 to $31.6 \%(273 / 864)$ after 2018 . Axillary sentinel lymph node biopsy in the CNB $(57.1 \%)$ or VABB $(77.9 \%)$ groups was significantly higher compared to the tumor resection biopsy and intraoperative frozen group $39.5 \%$ and $36.8 \%$, respectively). However, by using the double method tracing, some studies have reported that the successful rate of sentinel lymph node biopsy is $96 \%$ and the false negative rate is $10 \%$, while in the preoperative biopsy group, the successful rate of sentinel lymph node biopsy is $95 \%$ and the false negative rate is $5.6 \%$. It is believed that sentinel lymph node biopsy after breast tumor resection and biopsy is safe and feasible, but the false negative rate is slightly higher than that of the biopsy group (6). Puncture cytology can provide rapid confirmation of malignant diagnosis, and has been used as an early alternative to tumor resection and biopsy. However, the diagnostic performance of FNA varies according to the experience of operators and cell pathologists, and FNA shows a higher rate of nondiagnostic samples and false negative results (usually greater than $15 \%$ ) compared to $\mathrm{CNB}$, where false negative results are usually less than $5 \%(7,8)$. In 522 cases of breast cancer, FNA had a sensitivity of $68 \%$ and a specificity of $99.6 \%$, while CNB had a sensitivity of $90 \%$ and a specificity of $98.8 \%(9)$.

In a meta-analysis, FNA was reported to have a sensitivity of $74 \%$ and a specificity of $96 \%$, while ultrasound (US) or stereotactically guided CNB showed $87 \%$ sensitivity and $98 \%$ specificity (10). The results showed that FNA had a higher false negative rates and uncertainty $(8.9 \%)$, while in DCIS cases, the false negative rates were $5.1 \%$ and $2.3 \%$, respectively, and the pathological uncertainty rates were $8.1 \%$ and $9.1 \%$, respectively. However, in invasive breast cancer, the false negative and uncertainty rates were very low for both groups. The false negative rates of $\mathrm{CNB}$, local resection biopsy, and FNA were $2.3 \%, 0.3 \%$, and $9.1 \%$, respectively, and the rate of pathological uncertainty of these three biopsy methods was $1.3 \%, 1.8 \%$, and $13.6 \%$, respectively. It is suggested that the false negative of CNB is due to the inaccurate location of the focus, the insufficient amount of tissue obtained, and the puncture technique of the operator.

VABB technology was introduced into China in 1998, and the Food and Drug Administration (FDA) approved the complete resection of lesions in 2004. Since then, an increasing number of benign diseases indicated by imaging adopt minimally invasive circumcision instead of open breast segmental resection, resulting in a greater number 
of incidental cancer cases of "benign" breast diseases (BIRADS, Breast Imaging Reporting and Data System less than 4a) (11-15). VABB has been utilized in our hospital since 2009. The prevalence of incidental breast cancer detected by VABB ranged from $0.3 \%$ in the initial stage to $13.3 \%$ in the past 2 years. Furthermore, it has been reported that the learning curve time of VABB (4 weeks) is shorter than that of CNB (12 weeks) (16), and that there is a greater amount of tissue removed. Also, US-guided localization surgery is more suitable for in situ cancers, invasive cancers which need neoadjuvant therapy and more accurate evaluation of neoadjuvant therapy response (17-21). Compared to the negative predictive value and false negative rate of open surgical biopsy $(99.61 \%$ and $4.76 \%$ ), the negative predictive value of $\mathrm{VABB}$ was $99.77 \%$ and the false negative rate was $0.96 \%$ (22). These results demonstrated that VABB under the guidance of US exhibited the same diagnostic coincidence rate as that of segmental resection. There were no false negatives in 44 cases of carcinoma in situ and 110 cases of lesions less than $2 \mathrm{~cm}$. After extended resection of the tumor in one case of uncertain pathology, intraoperative freezing was also unclear, and was then confirmed by immunohistochemistry. At the same time, VABB had a higher breast-conserving rate $(57.1 \%$ vs. $41.8 \%)$ and sentinel lymph node biopsy rate (77.9\% vs. $39.5 \%$ ) compared to tumor resection. Recently, we also compared the medical economics of VABB and $\mathrm{CNB}$, which differed from the previous literature (23), and showed that the overall medical costs of the VABB group were lower than those of the CNB group (24).

At present, whether percutaneous tumor biopsy will cause tumor cells to be implanted in soft tissue or skin around the tumor remains debatable (1-3,25-29). Previous studies have reported that the positive rate of cytological examination of CNB syringe lavage fluid can reach 65\% (25), and pathological examination shows that the tumor implantation rate in the syringe can reach 50\% (26). Other studies have reported that the prognosis of patients that undergo biopsy is significantly lower than that of patients with tumor resection (27). Diaz et al. reported that $32 \%$ of resected specimens were implanted in the needle channel, with $21.6 \%$ in $1-2$ nests and $10.8 \%$ in multiple nests, while the tumor implantation rate in the CNB group was significantly higher than that of the VABB group (37\% vs. $23 \%, \mathrm{P}<0.05)(28)$. Uematsu et al. reported that the positive rate of VABB was $33 \%$, and was $69 \%$ in CNB (29). The follow-up study showed that the tumor implantation rate decreased gradually over time. The time from biopsy to tumor resection was less than 15 days, and the tumor cell implantation rate was $42 \%$, while the rate decreased to $15 \%$ when the interval was more than 28 days. It has been suggested that the cells implanted in the needle canal cannot survive for a long time (28). There were no reports of needle implantation in Michalopoulos et al.'s study (30). Thurfjell et al. reported 303 cases of clinically untouchable breast cancer, with a median follow-up time of 5.4 years and a local recurrence rate of $11 \%$. No radiotherapy was one of the causes of high recurrence (31), and the recurrence rate of radiotherapy was 3\% lower compared that with no radiotherapy (32). Also, the local recurrence rate was less than $4 \%$ when only extended local resection was performed without radiotherapy (32). More clinical studies have shown that biopsy is not correlated to the local recurrence rate and overall survival. Therefore, preoperative biopsy is recommended as the standard technique for the preoperative diagnosis of breast cancer $(4,33)$. According to the long-term follow-up prognosis data in this group, the preoperative biopsy mode had no effect on the prognosis of tumor-free and total survival time.

\section{Conclusions}

In summary, changes to the diagnosis and treatment of breast cancer have profoundly influenced and altered the methods of tumor biopsy. A more precise preoperative tumor biopsy mode was needed for comprehensive evaluation of patient information, especially pathological and molecular data, patients' participation in the whole treatment process, minimally invasive and sufficient tissue acquisition of the biopsy approach, and the repeatability of the biopsy. VABB biopsy will gradually become the main method of preoperative pathological evaluation of breast cancer. The overall success rate, sensitivity, and specificity of FNA, CNB, and VABB were not analyzed in this study. Due to the high overall success rate, multi-factor analysis was not conducted to identify the confounding factors of false negatives.

\section{Acknowledgments}

Funding: This study was supported by grants from the Zhejiang National Science Funding (no. LY18H160005) and the Zhejiang Medical Association Clinical Research Fund Project (no. 2019ZYC-A02). 


\section{Footnote}

Reporting Checklist: The authors have completed the STROBE reporting checklist. Available at http://dx.doi. org/10.21037/gs-21-7

Data Sharing Statement: Available at http://dx.doi. org/10.21037/gs-21-7

Conflicts of Interest: All authors have completed the ICMJE uniform disclosure form (available at http://dx.doi. org/10.21037/gs-21-7). The authors have no conflicts of interest to declare.

Ethical Statement: The authors are accountable for all aspects of the work in ensuring that questions related to the accuracy or integrity of any part of the work are appropriately investigated and resolved. This study was approved by Medical Ethics Committee of Sir Run Run Shaw Hospital, Comment (approval No. 2020-618-01). All procedures performed in this study involving human participants were in accordance with the Declaration of Helsinki (as revised in 2013). Individual consent for this retrospective analysis was waived.

Open Access Statement: This is an Open Access article distributed in accordance with the Creative Commons Attribution-NonCommercial-NoDerivs 4.0 International License (CC BY-NC-ND 4.0), which permits the noncommercial replication and distribution of the article with the strict proviso that no changes or edits are made and the original work is properly cited (including links to both the formal publication through the relevant DOI and the license). See: https://creativecommons.org/licenses/by-nc-nd/4.0/.

\section{References}

1. Fentiman IS, Millis RR, Chaudary MA, et al. Effect of the method of biopsy on the prognosis of and reliability of receptor assays in patients with operable breast cancer. $\mathrm{Br}$ J Surg 1986;73:610-2.

2. Liikanen J, Leidenius $M$, Joensuu $H$, et al. Breast cancer prognosis and isolated tumor cell findings in axillary lymph nodes after core needle biopsy and fine needle aspiration cytology: Biopsy method and breast cancer outcome. Eur J Surg Oncol 2016;42:64-70.

3. King TA, Hayes DH, Cederbom GJ, et al. Biopsy technique has no impact on local recurrence after breastconserving therapy. Breast J 2001;7:19-24.
4. Gradishar WJ, Anderson BO, Abraham J, et al. Breast Cancer, Version 3.2020, NCCN Clinical Practice Guidelines in Oncology. J Natl Compr Canc Netw 2020;18:452-78.

5. Davidson T, Ravid MM, Nissan E, et al.Correlations Between Core Needle Biopsy and Excisional Biopsy Findings in Suspected Breast Lesions: A Single Center Study. Isr Med Assoc J 2018;20:401-4.

6. Celebioglu F, Frisell J, Danielsson R, et al. Sentinel node biopsy in non-palpable breast cancer and in patients with a previous diagnostic excision. Eur J Surg Oncol 2007;33:276-80.

7. Shah VI, Raju U, Chitale D, et al. False-negative core needle biopsies of the breast: an analysis of clinical, radiologic, and pathologic findings in 27 concecutive cases of missed breast cancer. Cancer 2003;97:1824-31.

8. Pisano ED, Fajardo LL, Caudry DJ, et al. Fine-needle aspiration biopsy of nonpalpable breast lesions in a multicenter clinical trial: results from the radiologic diagnostic oncology group V. Radiology 2001;219:785-92.

9. Leifland K, Lagerstedt U, Svane G. Comparison of stereotactic fine needle aspiration cytology and core needle biopsy in 522 non-palpable breast lesions. Acta Radiol 2003;44:387-91.

10. Wang M, He X, Chang Y, et al. A sensitivity and specificity comparison of fine needle aspiration cytology and core needle biopsy in evaluation of suspicious breast lesions: A systematic review and meta-analysis. Breast 2017;31:157-66.

11. Klimberg VS, Rivere A. Ultrasound image-guided core biopsy of the breast. Chin Clin Oncol 2016;5:33.

12. Park HL, Kim KY, Park JS, et al. Clinicopathological Analysis of Ultrasound-guided Vacuum-assisted Breast Biopsy for the Diagnosis and Treatment of Breast Disease. Anticancer Res 2018;38:2455-62.

13. Li S, Wu J, Chen K, et al. Clinical outcomes of 1,578 Chinese patients with breast benign diseases after ultrasound-guided vacuum-assisted excision: recurrence and the risk factors. Am J Surg 2013;205:39-44.

14. Papathemelis T, Heim S, Lux MP, et al.Minimally Invasive Breast Fibroadenoma Excision Using an UltrasoundGuided Vacuum-Assisted Biopsy Device. Geburtshilfe Frauenheilkd 2017;77:176-81.

15. Lakoma A, Kim ES. Minimally invasive surgical management of benign breast lesions. Gland Surg 2014;3:142-8.

16. Zografos GC, Zagouri F, Sergentanis TN. Vacuumassisted breast biopsy: an easy-to-learn procedure?. Am J 
Surg 2008;196:798.

17. Nakano S, Imawari Y, Mibu A, et al. Differentiating vacuum-assisted breast biopsy from core needle biopsy: Is it necessary? Br J Radiol 2018;91:20180250.

18. Huang XC, Hu XH, Wang XR, et al. A comparison of diagnostic performance of vacuum-assisted biopsy and core needle biopsy for breast microcalcification: a systematic review and meta-analysis. Ir J Med Sci 2018;187:999-1008.

19. Park HL, Hong J. Vacuum-assisted breast biopsy for breast cancer. Gland Surg 2014;3:120-7.

20. Kuerer HM, Rauch GM, Krishnamurthy S, et al. A Clinical Feasibility Trial for Identification of Exceptional Responders in Whom Breast Cancer Surgery Can Be Eliminated Following Neoadjuvant Systemic Therapy. Ann Surg 2018;267:946-51.

21. Heil J, Richter H, Golatta M, et al. Vacuum-Assisted Biopsy to Diagnose a Pathological Complete Response in Breast Cancer Patients After Neoadjuvant Systemic Therapy. Ann Surg 2018;268:e60-1.

22. Tsai HY, Chao MF, Ou-Yang F, et al. Accuracy and outcomes of stereotactic vacuum-assisted breast biopsy for diagnosis and management of nonpalpable breast lesions. Kaohsiung J Med Sci 2019;35:640-5.

23. 23.Lee CI, Wells CJ, Bassett LW. Cost minimization analysis of ultrasound-guided diagnostic evaluation of probably benign breast lesions. Breast J 2013;19:41-8.

24. Grady I, Vasquez T, Tawfik S, et al. Ultrasound-Guided Core-Needle Versus Vacuum-Assisted Breast Biopsy: A Cost Analysis Based on the American Society of Breast Surgeons' Mastery of Breast Surgery Registry. Ann Surg Oncol 2017;24:676-82.

25. Uematsu T, Kasami M. Risk of needle tract seeding of

Cite this article as: Teng $\mathrm{R}$, Wei Q, Zhou J, Dong M, Jin L, Hu W, Chen J, Wang L, Zhao W. The influence of preoperative biopsy on the surgical method in breast cancer patients: a single-center experience of 3,966 cases in China. Gland Surg 2021;10(3):1038-1045. doi: 10.21037/gs-21-7 breast cancer: cytological results derived from core wash material. Breast Cancer Res Treat 2008;110:51-5.

26. Hoorntje LE, Schipper ME, Kaya A, et al. Tumour cell displacement after 14G breast biopsy. Eur J Surg Oncol 2004;30:520-5.

27. Kong YC, Bhoo-Pathy N, O'Rorke M, et al. The association between methods of biopsy and survival following breast cancer: A hospital registry based cohort study. Medicine (Baltimore) 2020;99:e19093.

28. Diaz LK, Wiley EL, Venta LA. Are malignant cells displaced by large-gauge needle core biopsy of the breast? AJR Am J Roentgenol 1999;173:1303-13.

29. Uematsu T, Kasami M. The use of positive core wash cytology to estimate potential risk of needle tract seeding of breast cancer: directional vacuum-assisted biopsy versus automated core needle biopsy. Breast Cancer 2010;17:61-7.

30. Michalopoulos NV, Zagouri F, Sergentanis TN, et al. Needle tract seeding after vacuum-assisted breast biopsy. Acta Radiol 2008;49:267-70.

31. Thurfjell MG, Jansson T, Nordgren H, et al.Local breast cancer recurrence caused by mammographically guided punctures. Acta Radiol 2000;41:435-40.

32. Loughran CF, Keeling CR. Seeding of tumour cells following breast biopsy: a literature review. Br J Radiol 2011;84:869-74.

33. Eberth JM, Xu Y, Smith GL, et al. Surgeon influence on use of needle biopsy in patients with breast cancer: a national medicare study. J Clin Oncol 2014;32:2206-16.

(English Language Editor: A. Kassem) 